\title{
ACUTE PYOGENIC INFECTIONS OF THE SPINE IN CHILDREN
}

\author{
A. E. Pritchard and W. A. L. Thompson, Liverpool, England
}

The early diagnosis of acute pyogenic osteomyelitis of the spine is often difficult. In the early stages of the disease localising physical signs may be absent and radiographs may show no obvious abnormality. In the present study the cases of six children with acute vertebral osteomyelitis have been reviewed. Five were treated at the Alder Hey Hospital, Liverpool, and the sixth at the Robert Jones and Agnes Hunt Orthopaedic Hospital, Oswestry. Special attention has been given to clinical and radiographic features which may be helpful in diagnosis.

\section{CASE REPORTS}

Case 1-A girl aged eleven years was admitted in January 1946 with a history of five weeks` lower thoracic pain and fever followed by the formation of an abscess in the temporo-mandibular region. There was rigidity of the spine with tenderness over the upper lumbar spinous processes. Radiographs showed decalcification of the ninth and tenth thoracic vertebrae with diminution of the intervertebral space. Radiographs taken two weeks later showed a paraspinal shadow suggestive of abscess formation. The diagnosis of spinal osteomyelitis was made and immobilisation on a frame was started. Penicillin was given. The girl made a full recovery.

Case 2-A boy aged two years was admitted in October 1950 with a history of ten days' anorexia, fretfulness and limping. The spine was rigid and the boy would not sit up. Radiographs taken three months after admission showed destructive changes in the contiguous margins of the fourth and fifth lumbar vertebrae. The child was kept on a frame for five months and made a full recovery. When he was seen five years after the acute illness his spine appeared quite normal; radiographs showed vertebral sclerosis and persistent narrowing of the intervertebral space.

Case 3-A boy aged eight years was admitted to hospital in Aden in June 1955 with a history of three days' malaise, headache, fever and stiffness of the neck. A diagnosis of poliomyelitis was made. Lumbar puncture at first showed increase of protein and chlorides with 245 white cells per cubic millimetre. One month later the spinal fluid was turbid; staphylococcus aureus was found on culture. Treatment with aureomycin was started. The boy was transferred to the Alder Hey Hospital, Liverpool, three months after the onset of the disease. Radiographs (Fig. 1) showed erosion of the contiguous margins of the second, third and fourth lumbar vertebrae with narrowing of the intervertebral spaces. Immobilisation on a frame was started and was continued for two months. The boy recovered fully.

Case 4-A boy aged eight years was admitted in July 1955 with a history of ten days' limping and pain in the left hip. He showed a marked flexion deformity of the hip with tenderness in the left renal angle. Erythrocyte sedimentation rate was 95 millimetres in one hour. White blood cells were 15,000 per cubic millimetre. A presumptive diagnosis of perinephric abscess was made and penicillin was given. Radiographs taken one month after admission showed erosions of the contiguous borders of the first and second lumbar vertebrae (Fig. 2). Immobilisation on a frame was begun. Progress was good and eventual recovery complete.

Case 5-A girl aged eight years was admitted with suspected acute pyelitis in December 1957, having had four days of lower thoracic pain with radiation into the right loin. There was tenderness over the first lumbar vertebra and in the right renal angle and hypochondrium. There was also a scoliosis with spasm of the spinal muscles. Radiographs showed no abnormality. She developed a right pleural effusion after three weeks but it was not until six weeks after admission that radiographs of the spine (Fig. 3) confirmed the diagnosis of osteomyelitis of the twelfth thoracic vertebra. Treatment with penicillin and streptomycin was started a few days after admission because blood culture showed a growth of staphylococcus pyogenes. Immobilisation on a frame was started only after the diagnosis was confirmed in January 1958. The patient made a good recovery.

Case 6-A boy aged nine years was admitted in December 1958 with a four days' history of backache and fever. There was tenderness of the back at the thoraco-lumbar level both in the midline and on the left side, with spasm of the spinal muscles. There was also deep tenderness in the left loin. Erythrocyte sedimentation rate was 85 millimetres in one hour, white blood cells were 12,000 per cubic millimetre and blood culture showed a growth of staphylococcus aureus. Radiographs initially showed no abnormality. On a provisional diagnosis of a perinephric abscess penicillin and streptomycin were 


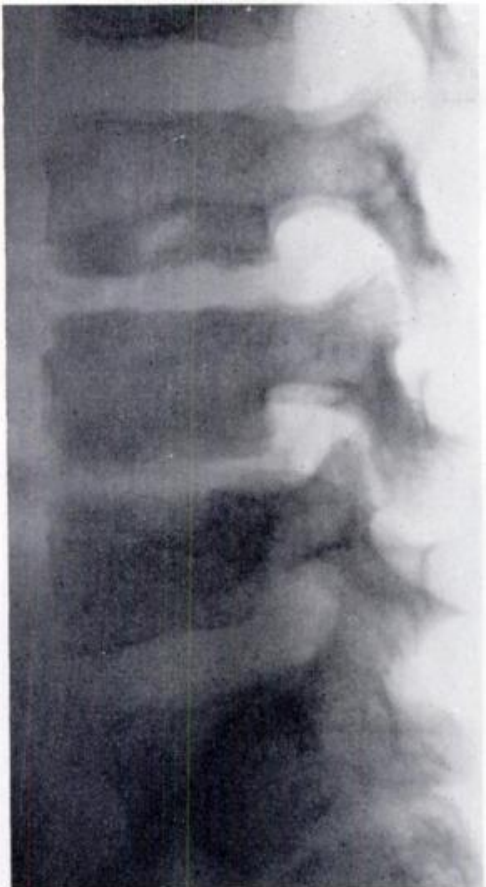

FIG. 1

Case 3-Radiograph taken three months after the onset of disease. showing erosion of the second, third and fourth lumbar vertebrae with narrowing of the intervertebral spaces.

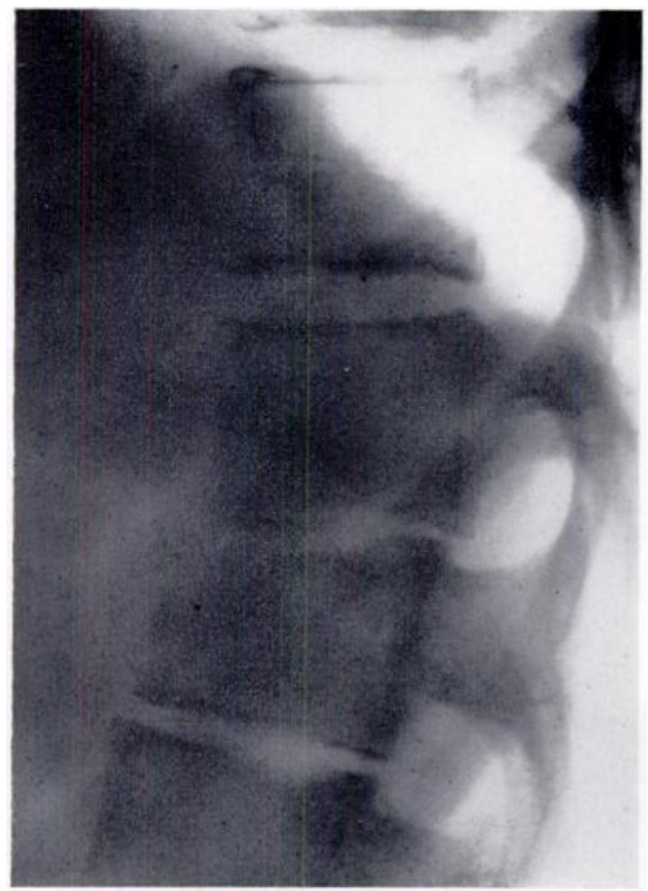

FIG. 3

Case 5-Radiograph taken six weeks after the onset of disease, showing destruction of the upper part of the body of the twelfth thoracic vertebra with loss of intervertebral space.

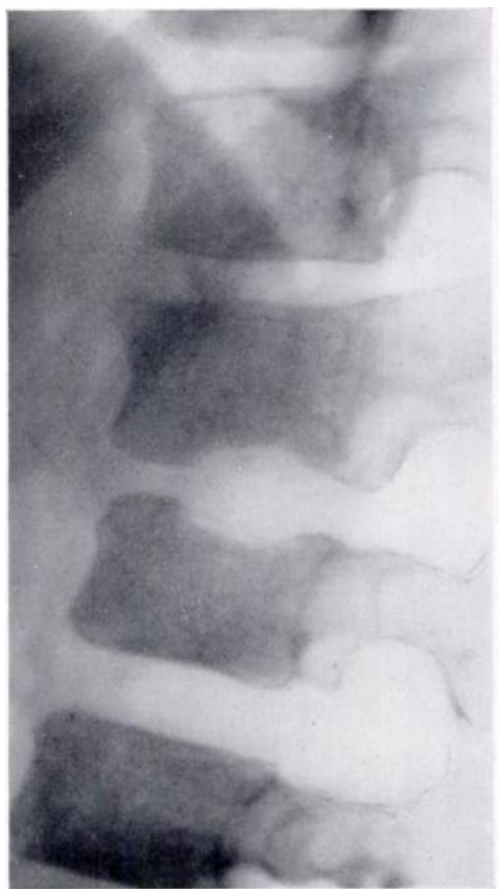

Fig. 2

Case 4-Radiograph taken six weeks after the onset of disease, showing erosions of the bodies of the first and second lumbar vertebrae.

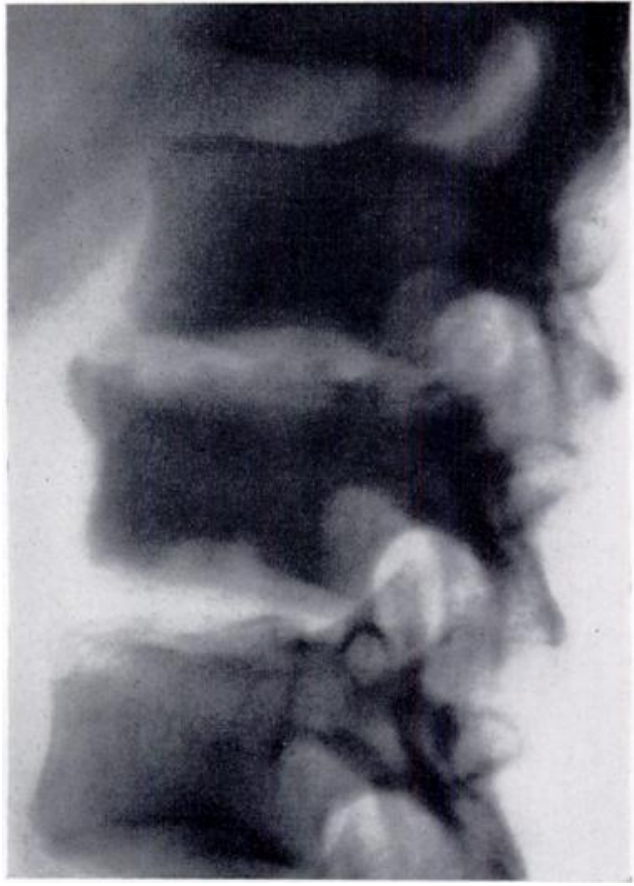

FIG. 4

Case 6-Radiograph taken six months after admission, showing condition in the phase of healing. Note the sclerosis and reduction of intervertebral space.

VOL. 42 B, NO. 1, FEBRUARY 1960

$\mathrm{F}-2_{(8)}$ 
given, and it was not until one month after admission that radiographs confirmed the diagnosis of osteomyelitis of the second lumbar vertebra. Progress was good and full recovery occurred. The radiological appearances in the healing phase six months after admission are shown in Figure 4.

\section{ANALYSIS OF CASES}

Clinical features-The onset is acute, with high temperature and toxaemia. Backache is complained of but is poorly localised; depending on the level of the lesion the pain may radiate into the chest or abdomen or occasionally into one of the limbs. The spinal muscles are in spasm and there may be a scoliosis. There may be a flexion contracture of the hip joint due to irritation of the psoas. In one patient (Case 3) stiffness of the neck with malaise and high temperature led to an initial diagnosis of acute anterior poliomyelitis. There was evidence of meningeal inflammation in this case.

There is tenderness in the erector spinae muscles and pain on percussion of the vertebrae involved and those adjacent to them. If there is an abscess the pus usually spreads anteriorly and laterally. If the infection is in the thoraco-lumbar region the lateral spread of pus may suggest a subphrenic or perinephric abscess, whereas a lower thoracic focus may produce a pleural effusion. The presumptive diagnosis of perinephric abscess was in fact made in two of our patients (Cases 4 and 6). Later a kyphos may develop.

Special investigations-Blood culture is usually positive in the initial pyrexial stage. The erythrocyte sedimentation rate is markedly raised, being between 60 and 120 millimetres in the hour, and the white cell count is raised to 15,000 or above. A negative Mantoux test has been very significant; a positive reaction is not of value.

Radiographic examination may show elevation of one or both sides of the diaphragm and perhaps an abscess shadow suggestive of subphrenic pus. The spine is radiologically normal at first, but after about three weeks, or earlier in very young children, areas of destruction appear on the contiguous margins of the involved vertebrae (Fig. 2), together with patchy osteoporosis of their bodies. The crescentic vertebral erosions appear rather behind the midline of the bodies. Later the intervertebral space narrows, and finally, in the healing phase, there is dense sclerosis of the adjacent margins of the vertebrae (Fig. 4).

Treatment-The clinical picture is clear enough to justify antibiotic treatment on suspicion in the early stages. After the third week radiographs permit a firm diagnosis. Antibiotics should be given for at least a month, and immobilisation (by frame or plaster bed) should be instituted as soon as possible, and continued for two to three months depending on progress. These measures yielded satisfactory results in our series even though they were begun late in each case because of delay in diagnosis. All our patients recovered completely, though the narrowing of the disc space is permanent. We cannot say whether or not this leads to backache in later life.

\section{DISCUSSION}

A study of the literature indicates that vertebral osteomyelitis has been recognised for a very long time. In 1931 Smith demonstrated the existence of blood vessels in the discs. He quotes Schmorl, " infectious disease leads to a diminished transparency and thickness of the discs. When the vertebral spongiosa is inflamed the disc exerts pressure which causes a collapse of the cancellous bone. The discs themselves later become inflamed, vascularised and shrunken from fibrotic and degenerative changes or may ossify."

Wilensky in 1929 and Kulowski in 1935 described the condition. Stammers (1938) described epidural suppuration as a complication. Turner (1938) stressed the difficulty of diagnosis and said that the condition should always be considered when abscesses formed around the kidney.

Butler, Blusger and Perry (1941) studied thirteen patients admitted to the London Hospital between 1921 and 1941, all of whom died. 
Guri (1946) in an excellent review discussed the "hip syndrome" with which these children often present, and described the pathology and clinical course of the disease in detail.

Bremner and Neligan (1953) described the condition in seven patients between the ages of nine months and two and a half years. The initial diagnoses were Pott's disease, "painful limp." encephalitis, " infected hip," urinary infection, "? sacro-iliac infection." " abdominal pain ? cause." All eventually responded well to treatment on a plaster bed and only one was treated with penicillin. Mantoux test was negative in all. In four there was spasm of the psoas. The radiographic findings and course of the disease were characteristic though the progress of these patients was less stormy than that of ours.

The site of the vertebral erosions corresponds to the position of the nucleus pulposus in the intervertebral disc. Their position raises the question of whether the inflammatory changes start in the nucleus and spread thence causing necrosis of the vertebrae by pressure.

\section{SUMMARY}

1. Six children suffering from acute infections of the spine have been studied.

2. Clinical and radiographic features are described. Reasons are given for bearing the condition in mind when dealing with cases of pyrexia of unknown origin in children.

3. Treatment is broadly outlined.

4. Radiographic findings are discussed in relation to the pathology of the disease.

We are grateful to Mr F. C. Dwyer, Mr A. M. Hendry and Mr G. L. Shatwell for permission to publish details of their patients.

We would also like to thank Professor B. L. McFarland for his help and advice, and Dr E. W. McMillan for his assistance with anatomical matters.

\section{REFERENCES}

Bremner, A. E., and Neligan, G. A. (1953): Benign Form of Acute Osteitis of the Spine in Young Children. British Medical Journal, i, 856.

Butler, E. C. B., Blusger, I. N., and Perry, K. M. A. (1941): Staphylococcal Osteomyelitis of the Spine. Lancet, i, 480.

GuRI, J. P. (1946): Pyogenic Osteomyelitis of the Spine. Journal of Bone and Joint Surgery, 28, 29.

Kulowski, J. (1935): Unusual Pyogenic Osteomyelitis. Journal of the Missouri State Medical Association, $32,85$. Smith. N. R. (1931): The Intervertebral Discs. British Journal of Surgery, 18, 358.

Stammers, F. A. R. (1938): Spinal Epidural Suppuration, with Special Reference to Osteomyelitis of the Vertebrae. British Journal of Surgery, 26, 366.

Turner, P. (1938): Acute Infective Osteomyelitis of the Spine. British Journal of Surgery, $26,71$.

Wilensky, A. O. (1929): Osteomyelitis of the Vertebrae. Annals of Surgery, 89, 561, 731. 